\title{
Is There a Link Between Carotid Atherosclerosis and Idiopathic Overactive Bladder Among Women with Metabolic Syndrome?
}

This article was published in the following Dove Press journal: Research and Reports in Urology

\author{
Gustavo Teixeira Fulton Schimit ${ }^{\prime}$ \\ Emerson Pereira Gregorio ${ }^{2}$ \\ Marcio Augusto Averbeck ${ }^{3}{ }^{3}$ \\ Matheus Junges de Souza ${ }^{4}$ \\ Acsa Caroline Mesquita \\ da Silva ${ }^{4}$ \\ Danielle Romano Tavares (D) $^{4}$ \\ Silvio Henrique Maia \\ de Almeida $\left.{ }^{5}\right)^{5}$ \\ 'Department of Surgery, Londrina State \\ University, Londrina, Brazil; ${ }^{2}$ School of \\ Medicine, Pontifical Catholic University \\ of Paraná, Paraná, Brazil; ${ }^{3}$ Video- \\ Urodynamics Unit, Moinhos de Vento \\ Hospital, Porto Alegre, Brazil; ${ }^{4}$ State \\ University of Londrina, Londrina, Brazil; \\ ${ }^{5}$ Discipline of Urology, Department of \\ Surgery, State University of Londrina, \\ Londrina, Brazil
}

\begin{abstract}
Purpose: To evaluate the association between increased intima-media thickness (IMT) and atherosclerotic carotid plaque $(\mathrm{CP})$ with idiopathic overactive bladder syndrome $(\mathrm{OAB})$ in women with metabolic syndrome (MetS).

Methods: This is a cross-sectional study, which included consecutive women aged 40-75 years with MetS, seeking medical assistance at a reference center between April and December 2016. OAB-V8 questionnaire was used to estimate the prevalence of OAB symptoms, which were defined by a score $\geq 8$ points. All patients underwent bilateral carotid artery ultrasound to assess IMT and CP. Atherosclerosis was defined by the identification of CP during ultrasound, which was diagnosed according to the Mannheim carotid intimamedia thickness and plaque consensus.

Results: Forty-five women were prospectively included. Mean age was $60 \pm 9.3$ years (range $40-75$ ys). Eighteen (40\%) patients were diagnosed with OAB. IMT in the general population was $0.72 \mathrm{~mm}(\mathrm{SD}=0.20)$. Overall prevalence of atherosclerosis, defined by the presence of the carotid artery plaque, was $51 \%$. OAB prevalence among women with atherosclerosis was higher than in those without atherosclerosis (56.52\% versus $22.73 \%)$, with a prevalence ratio of $2.49(\mathrm{p}=0.04)$. Additionally, OAB was associated with degree of carotid stenosis $(\mathrm{p}=0.029)$.
\end{abstract}

Conclusion: In this cohort of female patients with MetS, there was an association between carotid atherosclerosis and OAB. Identification of carotid ultrasound abnormalities may lead to refined treatment decision-making among OAB patients.

Keywords: carotid, atherosclerosis, ultrasound, lower urinary tract symptoms, overactive bladder

\section{Introduction}

According to the International Continence Society (ICS), idiopathic overactive bladder syndrome (OAB) is defined as urinary urgency, usually accompanied by frequency and nocturia, with or without urgency urinary incontinence, in the absence of urinary tract infection or other obvious pathology. ${ }^{1}$ OAB prevalence increases with age, affecting men and women similarly. ${ }^{2-4}$ Its etiology is not well known but is probably multifactorial, and there is increasing evidence for an association between $\mathrm{OAB}$ and cardiovascular risk factors, such as metabolic syndrome (MetS), smoking, and sedentary lifestyle. Additionally, clinical and experimental studies have assessed the effects of chronic bladder ischemia secondary to atherosclerosis in the etiology of OAB. ${ }^{3-8}$

Atherosclerosis causes occlusion of major arteries and smaller branches, which can impair the perfusion of multiple organs, including the urinary bladder. ${ }^{2}$ Hypoxia-induced
Correspondence: Marcio Augusto

Averbeck Rua Tiradentes, 333, 6th Floor

Porto Alegre, RS 90560-030, Brazil

Tel +55 5I 33I4 2940

Fax +55 5I 33142982

Email marcioaverbeck@gmail.com 
oxidative stress has been related to neural dysfunction and increased collagen content in the bladder wall. ${ }^{9,10}$

Inaba et al demonstrated that cardiovascular risk factors are associated with increased intima-media thickness (IMT) of the common carotid artery on vascular ultrasound. ${ }^{11}$ Measures of common carotid artery (CCA) subclinical atherosclerosis, such as intima-media thickness (IMT), adventitial diameter (AD), and carotid plaque (CP), are clinically important predictors of future CVD events. CP, which can also be effectively detected by vascular ultrasound, is a manifestation of atherosclerosis and appears to be a stronger cardiovascular risk predictor in comparison to IMT measurements alone. ${ }^{12,13}$

MetS is regarded as a marker of higher cardiovascular risk. However, the relationship between carotid artery changes, which can be evaluated by distinct ultrasound parameters, and OAB symptoms in women with MeTS has not been properly investigated so far. On the other hand, although women with MeTS have a higher prevalence of $\mathrm{OAB}$, not all will develop such syndrome. In this context, patient phenotyping may play a role in future preventive strategies.

This study aimed to evaluate the association between increased intima-media thickness (IMT) and atherosclerotic $\mathrm{CP}$ with idiopathic $\mathrm{OAB}$ in women with MetS.

\section{Materials and Methods Study Design}

This cross-sectional study was performed at a reference center (University Hospital) between April and December 2016 after receiving approval from local ethics committee (Research Ethics Committee of Universidade Estadual de Londrina protocol 1252631). The study was performed in accordance with the Declaration of Helsinki and informed consent was obtained from all included patients. Women aged 40-75 years diagnosed with MetS, and treated at a specialized outpatient clinic for endocrinology and vascular surgery, were prospectively enrolled.

MetS was defined according to the NCEP-ATP III criteria. ${ }^{14}$ At least three of the following five factors were needed to establish the diagnosis of MetS: 1) fasting glucose $\geq 100 \mathrm{mg} / \mathrm{dL}$ or use of medication for glucose intolerance; 2 ) blood pressure $\geq 130 / 85 \mathrm{mmHg}$ or use of medication for arterial hypertension (AH); 3) high-density lipoprotein cholesterol level $<50 \mathrm{mg} / \mathrm{dL}$; 4) triglycerides $\geq 150 \mathrm{mg} / \mathrm{dL}$ or use of medication for hypertriglyceridemia; and 5) abdominal obesity (abdominal circumference $>88 \mathrm{~cm}$ ).
At admission, a comprehensive urological and urogynecological history was taken, pelvic examination was performed, and urinary infections were ruled out. Exclusion criteria included: diagnosis of or previous treatment for lower urinary tract disease; pelvic organ prolapse; use of drugs affecting the lower urinary tract, including alpha blockers, $\beta 3$-adrenoceptor agonist, antimuscarinics (and medications with anticholinergic burden, such as tricyclic antidepressants, antihistamines, antiparkinsonism drugs, antipsychotics); psychiatric disorders; neurological disease; atherosclerotic disease with hemodynamic repercussion in the aortoiliac territory.

The "Overactive Bladder-Validated 8-question Screener" (OAB-V8) was used to estimate the prevalence of $\mathrm{OAB}$ symptoms, which were defined by a score $\geq 8$ points. ${ }^{15}$

\section{Carotid Artery Ultrasound}

Eligible patients were referred for carotid artery ultrasound. No patients declined study and no patient was withdrawn from protocol. A single trained researcher (specialized radiologist), who was blinded for patient's previous clinical assessments, performed all ultrasound examinations. Vascular ultrasound was carried out in compliance with international guidelines, using a high-frequency linear transducer (12 MHz) with Doppler color imaging (Esaote MyLab $\left.{ }^{\text {TM }} 50\right) .{ }^{16,17}$ Ultrasound characterization of the IMT was performed with the blood vessel in a longitudinal section and the walls aligned to form a double-line pattern with lumen-intima and tunica media-adventitia interfaces. The point for measurement of the carotid artery was $2 \mathrm{~cm}$ proximal to the bifurcation. The distance between the two acoustic interfaces in the posterior wall of the distal third of the common carotid artery was considered the IMT measurement in centimeters. Ultrasound assessments were performed bilaterally and repeated twice for determination of mean values, which were then compared to reference values. ${ }^{16-18}$

Atherosclerosis was defined by identification of $\mathrm{CP}$ during ultrasound, which was diagnosed according to the North American Symptomatic Carotid Endarterectomy Trial (NASCET) method and Mannheim carotid intimamedia thickness and plaque consensus, as a focal structure that encroaches into the arterial lumen of at least $0.5 \mathrm{~mm}$ and/or measuring $>50 \%$ of the value of the adjacent IMT measurement and/or an IMT measurement $>1.5 \mathrm{~mm} .{ }^{16-18}$ Degree of carotid stenosis was defined by color duplex with Doppler frequency shift and B-mode imaging, according to international guidelines. ${ }^{16-18}$ 


\section{Statistical Analysis}

MedCalc for Windows version 9.5.2.0 (MedCalc Software, Mariakerke, Belgium) was used and analysis was performed considering $\mathrm{p}<0.05$ as statistically significant and with $95 \%$ confidence interval (CI). Differences between the two groups $(\mathrm{OAB} \mathrm{V} 8 \geq 8$ or $\mathrm{OAB} \mathrm{V} 8<8)$ were evaluated by the $t$-test for independent samples and by the test of Chi-square for categorical variables. For dichotomous categorical variables, the prevalence ratio and the CI were also calculated.

A multivariate logistic regression analysis model was used to assess the association of different variables (age, IMT, degree of carotid stenosis and presence of the CP) with $\mathrm{OAB}$ symptoms. As a method for selecting the variables, stepwise regression was used, considering $\mathrm{p}<0.05$ as significant, and removing the model variable when $\mathrm{p}>0.20$.

\section{Results}

From April 2016 to December 2016, 45 consecutive patients were included. Mean age was $60 \pm 9.3$ years (range 40-75 ys). Eighteen (40\%) patients were diagnosed with $\mathrm{OAB}(\mathrm{OAB} \mathrm{V8} \geq 8)$. There was no difference between groups with or without $\mathrm{OAB}$ in terms of baseline characteristics, including age, BMI, comorbidities, cardiovascular risk factors and distribution of individual components of MetS (Table 1).

IMT in the general population was $0.72 \mathrm{~mm}(\mathrm{SD}=0.20)$. Degree of carotid stenosis (\%) in OAB patients showed higher medians, than those without OAB $(p=0.02)$. Overall prevalence of atherosclerosis, defined by the presence of the $\mathrm{CP}$, was $51 \%$. OAB prevalence among women with atherosclerosis was higher than in those without atherosclerosis $(56.52 \%$ versus $22.73 \%$ ), with a prevalence ratio of $2.49(\mathrm{p}=0.04)$. The multivariate analysis confirmed these results (odds ratio $=4.42 ; 95 \% \mathrm{CI}=1.21-16.11 ; \mathrm{p}=0.02$ ).

Ultrasound findings are presented in Table 2.

\section{Discussion}

This pilot study demonstrated an association between carotid artery ultrasound findings and OAB symptoms among patients with MetS. A statistically significant association has been found between OAB (determined by an OAB-V8 score $\geq 8$ points) and carotid artery atherosclerosis.

A few studies have previously evaluated the association between carotid artery ultrasound findings and OAB. Yeniel et al assessed women with $\mathrm{OAB} v s$ controls and demonstrated a link between carotid and femoral artery intima-media
Table I Baseline Patient Characteristics and Stratification According to OAB V8 Score ${ }^{a}$

\begin{tabular}{|c|c|c|c|c|}
\hline Variable & Total & OAB V8 $\geq 8$ & OAB V8 $<8$ & $P$ value \\
\hline & $n=45(100 \%)$ & $\mathrm{n}=18(40 \%)$ & $n=27(60 \%)$ & \\
\hline Age (years) & & & & \\
\hline Mean (SD) & 58.47 (7.7I) & $60.61(9.33)$ & $57.04(6.21)$ & $0.12 *$ \\
\hline BMI $\left(\mathrm{kg} / \mathrm{m}^{2}\right)$ & & & & \\
\hline Mean (SD) & $34.69(6.16)$ & $34.63(5.90)$ & $34.72(6.43)$ & $0.96 *$ \\
\hline$A C(\mathrm{~cm})$ & & & & \\
\hline Mean (SD) & $109.74(13.39)$ & $108.25(11.03)$ & I $10.74(14.88)$ & $0.54 *$ \\
\hline AMI/RM & & & & \\
\hline Yes & 4 (9.1 I\%) & I (25.00\%) & $3(75.00 \%)$ & $0.91 * *$ \\
\hline No & 4I (88.89\%) & 17 (41.46\%) & $24(58.54 \%)$ & \\
\hline $\mathbf{A H}$ & & & & \\
\hline Yes & 42 (93.33\%) & $17(40.48 \%)$ & $25(59.52 \%)$ & $0.71 * *$ \\
\hline No & $3(6.67 \%)$ & I (33.33\%) & 2 (66.67\%) & \\
\hline DM & & & & \\
\hline Yes & 34 (75.56\%) & II (32.35\%) & $23(67.65 \%)$ & $0.13 * *$ \\
\hline No & II (24.44\%) & $7(63.64 \%)$ & 4 (36.36\%) & \\
\hline Smoking & & & & \\
\hline Yes & $6(13.33 \%)$ & $2(33.33 \%)$ & $4(66.67 \%)$ & $0.92 * *$ \\
\hline No & 39 (86.67\%) & 16 (41.03\%) & 23 (58.97\%) & \\
\hline Dyslipidemia & & & & \\
\hline Yes & 4| (88.89\%) & $16(39.02 \%)$ & $25(60.98 \%)$ & $0.91 * *$ \\
\hline No & 4 (9.1 I\%) & $2(50.00 \%)$ & $2(50.00 \%)$ & \\
\hline
\end{tabular}

Notes: ${ }^{a}$ For dichotomous variables (yes/no), percentages are provided in parentheses in each of the three columns (total population, $O A B$ V8 $\geq 8, O A B$ V8 $<8$ ). *T Test and **Chi-square test.

Abbreviations: $O A B$, idiopathic overactive bladder syndrome; $O A B-V 8$, Overactive Bladder-Validated 8-Question Screener; BMI, body mass index; AC, abdominal circumference; AMI/RM, acute myocardial infarction/ revascularization of the myocardium; $\mathrm{AH}$, arterial hypertension; DM, diabetes mellitus.

Table 2 Carotid Artery Ultrasound Findings Stratified According to the OAB V8 Score ${ }^{a}$

\begin{tabular}{|l|l|l|l|l|}
\hline \multirow{2}{*}{ Variable } & Total & OAB V8 $\geq 8$ & OAB V8 $<8$ & \multirow{2}{*}{ p value } \\
\cline { 2 - 4 } & $\mathbf{n}=\mathbf{4 5}(\mathbf{1 0 0 \% )}$ & $\mathbf{n}=\mathbf{1 8}(\mathbf{4 0} \%)$ & $\mathbf{n = 2 7}(\mathbf{6 0 \%})$ & \\
\hline $\begin{array}{l}\text { IMT (mm) } \\
\text { Mean (SD) }\end{array}$ & $0.72(0.20)$ & $0.76(0.22)$ & $0.68(0.18)$ & $\mathrm{P}=0.19^{*}$ \\
\hline $\begin{array}{l}\text { Stenosis (\%) } \\
\text { Mean (SD) }\end{array}$ & $14.04(15.79)$ & $20.28(15.62)$ & $9.89(14.75)$ & $\mathrm{P}=0.02^{* *}$ \\
\hline $\begin{array}{l}\text { CP } \\
\text { Yes (51.I1\%) } \\
\text { No (48.89\%) }\end{array}$ & $22(51.11 \%)$ & $13(56.52 \%)$ & $10(43.48 \%)$ & $\begin{array}{l}\mathrm{P}=0.04 * * * \\
\mathrm{PR}=2.49 \\
\mathrm{Cl}=1.06-5.82\end{array}$ \\
\hline
\end{tabular}

Notes: ${ }^{a}$ For the dichotomous variable Carotid plaque (yes/no), percentages are provided in parentheses in each of the three columns (total population, $O A B \vee 8 \geq 8$, OAB V8 < 8). *T Test; **Mann-Whitney U-Test; ****Chi-square Test.

Abbreviations: OAB-V8, Overactive Bladder-Validated 8-Question Screener; IMT, Intima-media thickness; CP, carotid plaque; SD, standard deviation; IR, interquartile range; $\mathrm{PR}$, prevalence ratio; $\mathrm{Cl}$, confidence interval. 
thickness, OAB severity, and impaired vascular bladder perfusion. ${ }^{9}$ Uzun et al carried out a cross-sectional study to investigate carotid artery IMT in patients with LUTS/OAB. ${ }^{19}$ $\mathrm{AH}$, diabetes mellitus (DM), and CVD were exclusion criteria. Patients with LUTS had a significantly higher IMT than controls. Similarly, Takahashi et al and Azab evaluated nondiabetic men with LUTS and divided them into groups with and without atherosclerosis. ${ }^{20,21}$ Both identified an association between atherosclerotic disease and bladder dysfunction. Additionally, Lee et al demonstrated that the presence of $\mathrm{CP}$ in men was associated both with LUTS and erectile dysfunction. $^{22}$

Our research was different from previously published studies and had several strengths. Firstly, we have only included women with a MetS diagnosis according to the NCEP-ATP III criteria, opposed to previous studies, which excluded patients with DM. ${ }^{19-21}$ Identification of patients with MetS is particularly relevant, since it is regarded as a strong risk factor for cardiovascular outcomes. Another important point was the use of plaque definitions recommended by Mannheim Carotid Intima-Media Thickness and Plaque Consensus. ${ }^{20-22}$ Our study followed this consensus statement, which precisely establishes the difference between IMT and CP, homogenizing the criteria, facilitating data collection, interpretation, and comparing study results. ${ }^{17}$ In contrast to this, previous studies did not evaluate several atherosclerotic ultrasound parameters, sometimes limiting their analysis to IMT. ${ }^{19}$

There is a growing recognition of atherosclerotic plaque as a relevant finding, since plaque stability and vulnerability (eg, intraplaque hemorrhage, plaque ulceration, or fissuring) are more important than degree of stenosis in determining the risk of complications. ${ }^{23}$ With a rigorous ultrasound methodology, our proof-of-concept study suggests that CP is associated with OAB symptoms in patients with MetS. Thus, our findings add new insights to the current literature, showing that the presence of $\mathrm{CP}$ (and not just intimal stenosis) is related to $\mathrm{OAB}$. Thickening of the carotid wall is not synonymous with atherosclerosis, but it represents a subclinical vascular disease and is considered a marker of cardiovascular disease (CVD), which remains the most common cause of mortality in women worldwide. Subclinical atherosclerosis is intimately related to the onset of clinically apparent CVD and to CVD mortality, typically preceding the occurrence of clinical CVD by years to decades. ${ }^{12,24}$ Since stroke is a leading cause of death among women, any clinical factors that could help identify patients at increased risk are relevant and may allow early treatment and preventive measures. ${ }^{25}$
Pelvic arterial insufficiency may play an important role in the development of bladder dysfunctions such as OAB syndrome. Experimental models of pelvic arterial insufficiency resulted in ischemic conditions, which led to reduced bladder wall oxygen tension, increased oxidative stress and were associated with upregulation of oxidative stresssensitive genes, muscarinic receptor over-reactivity, ultrastructural damage, and neurodegeneration. ${ }^{5}$ Clinical assessment of atherosclerosis and endothelial dysfunction may be of relevance for future research initiatives, in order to check if a drug may prevent progression of ischemiarelated functional and morphological bladder changes. ${ }^{5}$ More recently, it has been suggested that there are distinct subtypes of $\mathrm{OAB}$ and that identification of such phenotypes may lead to a personalized approach to therapy. ${ }^{26,27}$ This strategy may translate into refined treatment decisionmaking and better outcomes in the near future, ${ }^{27}$ for example, antimuscarinics, and botulinum toxin, have been demonstrated to be less effective in patients with metabolic syndrome, or at least less effective than in other patient populations. In contrast, the beta-3 adrenoreceptor agonist mirabegron, which has been described initially as an antiobesity drug, was found to be equally active in both obese and nonobese $\mathrm{OAB}$ patients. ${ }^{27}$ Besides overactive bladder syndrome, beta- 3 agonists may impact metabolic disease, insulin sensitivity, and triglyceride clearance. ${ }^{5}$

Personalizing the care of patients of MetS with OAB makes sense in a way that these patients can be motivated for lifestyle changes to alter modifiable risk factors. Previous studies suggested that vascular risk factors play a role in the development of LUTS in both sexes, with a negative impact on quality of life. ${ }^{28,29}$ Based on our pilot study findings, one could propose that women with Mets and carotid ultrasound abnormalities, but still without OAB symptoms, should be advised about the possible risk of developing lower urinary tract symptoms, strengthening the importance of behavioral changes. Logically, these findings are of explorative nature and need further validation.

Although our study protocol had strict inclusion criteria and followed international guidelines on carotid ultrasound assessments, our sample size was small (pilot study). Further research is still needed to confirm the association between carotid ultrasound abnormalities and $\mathrm{OAB}$, as well as the relationship with visceral adiposity, hormonal status ${ }^{30}$ and the implications in terms of future cardiovascular events among women with MetS. 


\section{Conclusions}

In this cohort of female patients with MetS, there was an association between carotid atherosclerosis and OAB. Identification of carotid ultrasound abnormalities may lead to a refined treatment decision-making among OAB patients.

\section{Disclosure}

The authors report no significant financial interest or potential conflict of interest relevant to this article.

\section{References}

1. Haylen BT, de Ridder D, Freeman RM, et al. An international urogynecological association (IUGA)/international continence society (ICS) joint report on the terminology for female pelvic floor dysfunction. Neurourol Urodyn. 2010;29:4-20. doi:10.1002/nau.20798

2. Coyne KS, Sexton CC, Thompson CL, et al. The prevalence of lower urinary tract symptoms (LUTS) in the USA, the UK and Sweden: results from the Epidemiology of LUTS (EpiLUTS) study. BJU Int. 2009;104:352-360. doi:10.1111/j.1464-410X.2009.08427.x

3. Irwin DE, Milsom I, Hunskaar S, et al. Population-based survey of urinary incontinence, overactive bladder, and other lower urinary tract symptoms in five countries: results of the EPIC study. Eur Urol. 2006;50:1306-1314. doi:10.1016/j.eururo.2006.09.019

4. Milsom I, Abrams P, Cardozo L, Roberts RG, Thüroff J, Wein AJ. How widespread are the symptoms of an overactive bladder and how are they managed? A population-based prevalence study. BJU Int. 2001;87:760-766. doi:10.1046/j.1464-410x.2001.02228.x

5. Andersson KE, Boedtkjer DB, Forman A. The link between vascular dysfunction, bladder ischemia, and aging bladder dysfunction. Ther Adv Urol. 2017;9:11-27. doi:10.1177/1756287216675778

6. Nomiya M, Sagawa K, Yazaki J, Takahashi N, Kushida N, Haga N. Increased bladder activity is associated with elevated oxidative stress markers and proinflammatory cytokines in a rat model of atherosclerosis-induced chronic bladder ischemia. Neurourol Urodyn. 2012;31:185-189. doi:10.1002/nau.v31.1

7. Wein AJ, Rackley RR. Overactive bladder: a better understanding of pathophysiology, diagnosis and management. J Urol. 2006;175:S510. doi:10.1016/S0022-5347(05)00313-7

8. Fitzgerald MP, Link CL, Litman HJ, Travison TG, McKinlay JB. Beyond the lower urinary tract: the association of urologic and sexual symptoms with common illnesses. Eur Urol. 2007;52:40715. doi:10.1016/j.eururo.2007.03.014

9. Yeniel AO, Ergenoglu AM, Meseri R, Kismali E, Ari A, Kavukcu G. Is overactive bladder microvasculature disease a component of systemic atherosclerosis? Neurourol Urodyn. 2017;37:1372-1379. doi:10.1002/nau.23452

10. Averbeck MA, De Lima NG, Motta GA, et al. Collagen content in the bladder of men with LUTS undergoing open prostatectomy: a pilot study. Neurourol Urodyn. 2018;37:1088-1094. doi:10.1002/nau. v37.3

11. Inaba Y, Chen JA, Bergmann SR. Carotid plaque, compared with carotid intima-media thickness, more accurately predicts coronary artery disease events: a meta-analysis. Atherosclerosis. 2012;220:128-133. doi:10.1016/j.atherosclerosis.2011.06.044

12. Keteepe-Arachi T, Sharma S. Underestimating risk in women delays diagnosis of CVD. Practitioner. 2016;260:11-15.

13. Murray CSG, Nahar T, Kalashyan H, Becher H, Nanda NC. Ultrasound assessment of carotid arteries: current concepts, methodologies, diagnostic criteria, and technological advancements. Echocardiography. 2018;35:2079-2091. doi:10.1111/echo.14197
14. Grundy SM, Cleeman JI, Daniels SR, Donato KA, Eckel RH, Franklin BA. Diagnosis and management of the metabolic syndrome: an American heart association/national heart, lung, and blood institute scientific statement. Circulation. 2005;17:2735-2752. doi:10.1161/ CIRCULATIONAHA.105.169404

15. Acquadro C, Kopp Z, Coyne KS, Corcos J, Tubaro A, Choo MS. Translating overactive bladder questionnaires in 14 languages. Urology. 2006;67:536-540. doi:10.1016/j.urology.2005.09.035

16. Stein JH, Korcarz CE, Hurst RT, Lonn E, Kendall CB, Mohler ER. Use of carotid ultrasound to identify subclinical vascular disease and evaluate cardiovascular disease risk: a consensus statement from the American society of echocardiography carotid intima-media thickness task force. Endorsed by the society for vascular medicine. $J \mathrm{Am}$ Soc Echocardiogr. 2008;21:93-111. doi:10.1016/j.echo.2007.11.011

17. Touboul PJ, Hennerici MG, Meairs S, Adams H, Amarenco P, Bornstein N. Mannheim carotid intima-media thickness and plaque consensus (2004-2006-2011). An update on behalf of the advisory board of the 3rd, 4th and 5th watching the risk symposia, at the 13th, 15th and 20th European Stroke Conferences, Mannheim, Germany, 2004, Brussels, Belgium, 2006, and Hamburg, Germany, 2011. Cerebrovasc Dis. 2012;34:290-296. doi:10.1159/000343145

18. Lorenz MW, von Kegler S, Steinmetz H, Markus HS, Sitzer M. Carotid intima-media thickening indicates a higher vascular risk across a wide age range: prospective data from the Carotid Atherosclerosis Progression Study (CAPS). Stroke. 2006;37:87-92. doi:10.1161/01. STR.0000196964.24024.ea

19. Uzun H, Çiçek Y, Kocaman SA, Durakoğlugil ME, Zorba OÜ. Increased pulse-wave velocity and carotid intima-media thickness in patients with lower urinary tract symptoms. Scand $J$ Urol. 2013;47:393-398. doi:10.3109/21681805.2013.780185

20. Takahashi N, Shishido K, Sato Y, Ogawa S, Oguro T, Kataoka M. The association between severity of atherosclerosis and lower urinary tract function in male patients with lower urinary tract symptoms. LUTS. 2012;4:9-13. doi:10.1111/j.1757-5672.2011.00 098.x

21. Azab S. The impact of atherosclerosis on lower urinary tract function. Aging Male. 2013;16:108-111. doi:10.3109/13685538. 2013.795532

22. Lee JH, Kim SK, Lee DG. Associations of carotid artery plaque with lower urinary tract symptoms and erectile dysfunction. Int Urol Nephrol. 2014;46:2263-2270. doi:10.1007/s11255-014-0830-y

23. Brinjikji W, Huston J 3rd, Rabinstein AA, Kim GM, Lerman A, Lanzino G. Contemporary carotid imaging: from degree of stenosis to plaque vulnerability. J Neurosurg. 2016;124:27-42. doi:10.3171/ 2015.1.JNS142452

24. Wang D, Jackson EA, Karvonen-Gutierrez CA, Elliott MR, Harlow SD. Healthy lifestyle during the midlife is prospectively associated with less subclinical carotid atherosclerosis: the study of women's health across the nation. J Am Heart Assoc. 2016;7: e010405. doi:10.1161/JAHA.118.010405

25. Girijala RL, Sohrabji F, Bush RL. Sex differences in stroke: review of current knowledge and evidence. Vasc Med. 2017;22:135-145. doi: $10.1177 / 1358863 X 16668263$

26. Apostolidis A, Averbeck MA, Sahai A, Rahnama'i MS, Anding R, Robinson D. Can we create a valid treatment algorithm for patients with drug resistant overactive bladder (OAB) syndrome or detrusor overactivity (DO)? Results from a think tank (ICI-RS 2015). Neurourol Urodyn. 2017;36:882-893. doi:10.1002/nau.23170

27. Peyronnet B, Mironska E, Chapple C, Cardozo L, Oelke M, Dmochowski R. A comprehensive review of overactive bladder pathophysiology: on the way to tailored treatment. Eur Urol. 2019;75: 988-1000. doi:10.1016/j.eururo.2019.02.038

28. Ponholzer A, Temml C, Wehrberger C, Marszalek M, Madersbacher S. The association between vascular risk factors and lower urinary tract symptoms in both sexes. Eur Urol. 2006;50(3):581-586. Epub 2006 Feb 17. doi:10.1016/j.eururo.2006.01.031 
29. Yeniel AO, Ergenoglu AM, Meseri R, Ari A, Sancar C, Itil IM. Systemic atherosclerosis and voiding symptom. Eur J Obstet Gynecol Reprod Biol. 2017;210:306-309. Epub 2017 Jan 9. doi:10.1016/j.ejogrb. 2017.01.005
30. Anık Ilhan G, Yıldızhan B. Visceral adiposity indicators as predictors of metabolic syndrome in postmenopausal women. Turk $J$ Obstet Gynecol. 2019;16(3):164-168. Epub 2019 Oct 10. doi:10.4274/tjod. galenos.2019.62558

\section{Publish your work in this journal}

Research and Reports in Urology is an international, peer-reviewed, open access journal publishing original research, reports, editorials, reviews and commentaries on all aspects of adult and pediatric urology in the clinic and laboratory including the following topics: Pathology, pathophysiology of urological disease; Investigation and

Submit your manuscript here: https://www.dovepress.com/research-and-reports-in-urology-journal treatment of urological disease; Pharmacology of drugs used for the treatment of urological disease. The manuscript management system is completely online and includes a very quick and fair peer-review system, which is all easy to use. Visit http://www.dovepress.com/ testimonials.php to read real quotes from published authors. 